\title{
Stability Control in a Supply Chain: Total Costs and Bullwhip Effect Reduction
}

\author{
Fernanda Strozzi ${ }^{*}$, , Carlo Noè ${ }^{1}$ and José-Manuel Zaldívar ${ }^{2}$ \\ ${ }^{I}$ Engineering Faculty, Carlo Cattaneo University (LIUC), Castellanza, Italy \\ ${ }^{2}$ European Commission, Joint Research Centre, Ispra, Italy
}

\begin{abstract}
The bullwhip effect refers to the phenomenon of demand distortion in a supply chain. By eliminating or controlling this effect, it is possible to increase product profitability. The main focus of this work is to apply a control technique, based on the divergence of system, to reduce the bullwhip effect in a single-product one echelon supply chain, in which an Order-Up-To (OUT) order policy is applied. First the relationships between bullwhip, stability of the supply chain and the total costs are analyzed. Second the divergence-based control strategy is applied to stabilize the supply chain dynamics with a considerable reduction of the total costs (>30\%) and, in relevant cases, of the bullwhip effect.
\end{abstract}

Keywords: Bullwhip effect, order-up-to policy, supply chain.

\section{INTRODUCTION}

The main objective of an optimal ordering policy is that of keeping close production and demand, while inventory levels and capacity requirements are kept at minimum levels [1]. However, a typical effect that arises in supply chains is the bullwhip effect. This effect refers to the phenomenon that occurs when orders to the supplier have larger variance than the ones from the customers, i.e. variance amplification [25].

The first academic description of the bullwhip phenomenon is usually ascribed to Forrester [6], who explained it as a lack of information looping between the components of the supply chain and by the non-linear interactions existing, which are difficult to deal with using managerial intuition. Sterman [7] simulated the bullwhip effect in the Beer Game model and confirmed these explanations. Other causes of bullwhip effect are [8-9]: overreaction to backlogs, errors in demand forecasting, neglecting to order in an attempt to reduce inventory, lack of communication and coordination up and down the supply chain, delay times for information and material flow and fluctuating prices. In $[8,9]$ several countermeasures were also presented.

The economic impact of bullwhip effect was studied in [10], who showed that by eliminating it, one could decrease the stock expenses around $15-30 \%$. For this reason, different bullwhip reduction techniques have appeared in literature. These techniques are based on improving demand forecast, improving the communication in the supply chain, applying a proportional controller to the order policy [1], filtering the demand in order to reduce the variability [11], replacing crisp orders by fuzzy numbers [3], applying Petri nets to improve the coordination of the supply chain [12], using

*Address correspondence to this author at the Engineering Faculty, Carlo Cattaneo University (LIUC), Castellanza, Italy; E-mail: fstrozzi@liuc.it genetic algorithm (GA) to optimize the order policy $[13,14]$. In [15] the impact of the centralization of the information was studied and it was shown that, even if an improvement of the coordination can decrease the bullwhip effect, it cannot eliminate it.

The distortion of the demand along a supply chain is in relationship with its parametric sensitivity. Local stability analysis for different types of complex supply chains was studied in [16], whereas Kleijnen [17] reviewed the role of sensitivity analysis in supply chain simulations. Non-linear behaviour, the relationships between global stability and damped oscillations, and the extraordinary complexity and the large variety of bifurcations have been studied in the Beer Game model [18-19]. Techniques to reduce the bullwhip effect based on considering the supply chain as a dynamical system and the application of control techniques have been recently summarized by Sarimveis et al. [20]. These control methodologies span from the application of a proportional controller $[1,21]$ to highly sophisticated techniques such as model predictive control [22].

In this work we will try to control the local stability of a simple supply chain in order to reduce the costs as well as the bullwhip effect. However, since the considered system is linear, local and global stabilities are the same and therefore, we will simply speak about stability. The supply chain considered is single-product, one echelon with a zero replenishment lead time and only a single, order-of-events, review period in which an Order-Up-To (OUT) order policy is applied and an auto regressive and moving average (ARMA) model is used to forecast the demand. Using this model the relationship between bullwhip and stability will be analysed, as well as the relationships between the divergence criterion and stability conditions. By stabilising the supply chain by controlling the order policy with the divergence of the system, we will show how it is possible to reduce the total costs and, in several cases, the bullwhip. The novelty of this control technique with respect to others techniques that have 
studied the relationship between total cost, bullwhip and stability in supply chains, is that this strategy can easily be extended on-line to provide an order policy without the knowledge of the system investigated. Using delayed vectors of a state space variable and applying non-linear dynamical systems techniques for the reconstruction of the divergence as in $[23,24]$, we would be able of detecting the stability properties of the chain and acting with a right order policy.

In this paper, we have developed the control strategy and assessed its performance using the analytical values of the divergence obtained from the model of the supply chain.

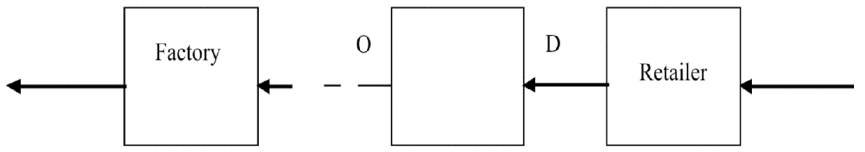

Fig. (1). Block diagram of a one echelon supply chain. The arrows indicate the direction of the Orders.

\section{THE SUPPLY CHAIN AND ORDER POLICY MODEL}

Let us consider a supply chain as represented in Fig. (1). The inventory is used to improve the service to the customers and to protect the production system from fluctuations in the demand. Different replenishment policies exist [25]. Two basic types of inventory replenishment rules are: fixed order and periodic re-ordering systems [26]. Fixed order systems result in the same quantity of product ordered at varying time intervals. In periodic systems a variable amount of products are ordered at fixed time intervals and the decision maker has to determine an Order-Up-To (OUT) level for each period. In this work OUT policy will be considered. Furthermore, following [5], we have chosen to model demand pattern as an ARMA stochastic process of order one. Gilbert [27] has shown that, when an independently and identically distributed ARMA demand pattern has passed through an OUT policy, it does not change the pattern. This implies that the results obtained studying the response of an ARMA demand pattern is relevant for any echelon of a supply chain.

Two of the most important processes, which are in a certain way entangled, are the ordering and the delivering of purchased items. The delays and the non-linearities in the supply chain model give rise to the bullwhip effect, i.e. the fluctuations in the orders that increase as the orders move up in the chain. The bullwhip is quantified as follows:

$$
\text { Bullwhip }=\frac{\operatorname{var}(O)}{\operatorname{var}(D)}
$$

where $O$ is the order to the supplier or to the production and $D$ is the customer demand.

\section{Costs Calculation}

Following [1], when the orders are higher than the capacity limit $K$, then ordering $\operatorname{cost} c_{0}$ is charged instead of $c$, see Eq. (2). This means that when the orders in each period are above the normal capacity limit, it is assumed that there is another source of supply. Examples of this alternative source of supply may include overtime working, purchasing or subcontracting. Holding and Shortage costs are calculated in accordance with Eqs. (3) and (4), where and $s$ and $h$ are the stock out costs and the inventory holding costs per unit per period, respectively. The sum of these costs yields the total cost per period, Eq. (5).

-Ordering costs $\left(O C_{t}\right)$ :

$O C_{t}=\left\{\begin{array}{l}c \cdot O_{t} \text { if } O_{t}<K \\ c \cdot K+c_{0} \cdot\left(O_{t}-K\right) \text { if } O_{t} \geq K\end{array}\right.$

- Holding costs $\left(H C_{t}\right)$ :

$H C_{t}= \begin{cases}h \cdot N S_{t} & \text { if } N S_{t}>0 \\ 0 & \text { if } N S_{t} \leq 0\end{cases}$

- Shortage costs $\left(S C_{t}\right)$ :

$S C_{t}= \begin{cases}s \cdot\left|N S_{t}\right| & \text { if } N S_{t}<0 \\ 0 & \text { if } N S_{t} \geq 0\end{cases}$

Finally, the total costs $\left(T C_{t}\right)$ are calculated as:

$T C_{t}=O C_{t}+H C_{t}+S C_{t}$

\section{Order Policy: OUT Policy}

The Order Up To (OUT) policy is frequently used as a standard ordering algorithm, in many material requirement planning (MRP) systems [24], to achieve customer service, inventory and capacity trade-off. At each time step, one reviews the inventory position and then places an "order" to bring it "-up-to" a defined level. In this case, the inventory level is reviewed at the beginning of the period and ordering decision is made. At the end of the period, the customer order is received and demand is formulated and fulfilled. Therefore, it takes one period to receive the order placed. Unmet demand in a period is backordered $[1,5]$. This OUT policy is able, in some cases, to minimize the inventory costs and the bullwhip [28].

The Order Up to Level, $S_{t}$, is updated every period according to the OUT policy:

$$
S_{t}=\bar{D}_{t}+k \sigma_{D_{t}}
$$

where $\overline{D_{t}}$ is the estimated demand at the end of period t, $\sigma_{D_{t}}$ is the standard deviation of the demand and $k$, the safety factor, is defined as:

$$
k=G^{-1}\left(\frac{s}{s+h}\right)
$$

where $G$ is the standard normal cumulative distribution,

$$
G(x)=G(x \mid \mu, \sigma)=\frac{1}{\sigma \sqrt{2 \pi}} \int_{-\infty}^{x} e^{\frac{-(t-\mu)^{2}}{2 \sigma^{2}}} d t
$$

The classical Order-Up-To policy definition is completed when net stock $N S_{t}$ is subtracted from inventory position:

$O_{t}=S_{t}-N S_{t}=\overline{D_{t}}+k \sigma_{D_{t}}-N S_{t}$

In accordance with $[1,5]$, a modification to the classical OUT policy was introduced to provide more freedom in 
shaping its dynamic response. They proposed to use a proportional controller by introducing a constant $l / T_{i}$ in the inventory position feedback loop as follows:

$O_{t}=\overline{D_{t}}+\frac{1}{T_{i}}\left(k \sigma_{D_{t}}-N S_{t}\right)$

Chen and Disney $[1,5]$ called this the modified OUT policy. The following equation completes the definition of $N S_{t}$ :

$$
N S_{t}=N S_{t-1}+O_{t-1}-D_{t-1}
$$

where $D_{t-1}$ is the real demand at time $t-1$.

\section{Demand Forecast}

In this work we will consider the demand to be a stochastic variable:

$$
D_{t}=\eta_{t}+\mu
$$

where $\eta_{t}$ is a random normal variable, with mean zero and unity variance, and $\mu$ is the mean of the demand.

Let us consider an ARMA process that can be written as:

$$
\bar{D}_{t}=\mu+\rho\left(\bar{D}_{t-1}-\mu\right)+\varepsilon_{t}+\theta \cdot\left(D_{t-1}-\bar{D}_{t-1}\right)
$$

where $\rho$ and $\theta$ are the model parameters with the initial condition $\bar{D}_{0}=\mu+\varepsilon_{0}$. The forecast error $\varepsilon_{t}$ is supposed to be a white noise process. In addition, we consider that $\varepsilon_{t-1}=D_{t-1}-\bar{D}_{t-1}$ has been actualized with the last known values and therefore is not a stochastic value.

Finally, the following numerical scenario will be considered. The average demand is $\mu=4$ units per period, the cost to produce a unit in normal production is $c=100 €$ per unit per period, and in overtime production is $c_{0}=200 €$ per unit per period. The inventory holding cost is $h=10 €$ per unit per period, the shortage cost is $s=50 €$ per unit per period and the capacity limit $K=12$ units per period. The inventory safety factor is set to $k \sigma_{D}=0.2 \mu[1,5]$.

\section{STABILITY AND BULLWHIP}

Let us consider, the dynamical system given by the Eqs. (10), (11) and (13), which represents one level of the supply chain of Fig. (1). These equations can be written in a matricial form as follows:

$$
\begin{gathered}
{\left[\begin{array}{c}
O_{t} \\
N S_{t} \\
\bar{D}_{t}
\end{array}\right]=\left[\begin{array}{ccc}
-1 / T_{i} & -1 / T_{i} & \rho-\theta \\
1 & 1 & 0 \\
0 & 0 & \rho-\theta
\end{array}\right]\left[\begin{array}{c}
O_{t-1} \\
N S_{t-1} \\
\bar{D}_{t-1}
\end{array}\right]+} \\
{\left[\frac{1}{T_{i}}\left(k \cdot \sigma+D_{t-1}\right)-\mu \cdot \rho+\theta \cdot D_{t-1}+\varepsilon_{t}+\mu\right.} \\
-D_{t-1} \\
-\mu \cdot \rho+\theta \cdot D_{t-1}+\varepsilon_{t}+\mu
\end{gathered}
$$

The matrix $(3 \times 3)$ on the right side is the Jacobian, $J$, of the system i.e. the matrix of all first-order partial derivatives.
The stability of the dynamics is given by the eigenvalues $\lambda_{1}, \lambda_{2}, \lambda_{3}$ of this matrix:

$J=\left[\begin{array}{ccc}-1 / T_{i} & -1 / T_{i} & \rho-\theta \\ 1 & 1 & 0 \\ 0 & 0 & \rho-\theta\end{array}\right]$

i.e. the solutions of the equation

$$
\operatorname{det}(J-\lambda I)=(\rho-\theta-\lambda)\left[\left(-\frac{1}{T_{i}}-\lambda\right)(1-\lambda)+\frac{1}{T_{i}}\right]=0
$$

which are

$$
\lambda_{1}=0, \lambda_{2}=\rho-\theta, \lambda_{3}=1-\frac{1}{T_{i}}
$$

The stability is ensured if all the eigenvalues are smaller than one in absolute value then $|\rho-\theta|<1$ which implies $-1<\rho-\theta<1$, and $\left|1-1 / \mathrm{T}_{\mathrm{i}}\right|<1$ and therefore $T_{i}>1 / 2$.

Stability means that a small change in the demand forecast $\left(\overline{D_{t}}\right)$ will produce a small change on the $\operatorname{Order}\left(O_{t}\right)$ or on the Net Stock $\left(N S_{t}\right)$ and the bullwhip cannot be too high.

Bullwhip measure, Eq. (1) considers only Order and Demand forecast and, hence, the variation of Net Stock is not taken into account. For this reason, it is not a global measure of the stability of the supply chain. In the specific case when $T_{i}=1$, and stability is given by the value of $\lambda_{2}$, the generated bullwhip surface when the parameters $\rho$ and $\theta$ are varying between -1 and 1 is showed in Fig. (2). The stability limits are indicated by the planes 1 and -1 , whereas $\lambda_{2}$ is represented by the inclined plane $\rho-\theta$. The stability region in the parameter space $(\rho, \theta)$ is the one inside the projection of the intersection of the surface $\rho-\theta$ with the surfaces planes $l$ and -1 . As we can see, this region contains even the parameter values for which bullwhip equals to 1 (intersections of bullwhip surface with surface plane 1) that means no bullwhip. Nevertheless it may occur that regions with bullwhip relatively small (for example for high values of $\rho$ and small values of $\theta$ ), are in the instability region, i.e. $\rho-\theta>1$.

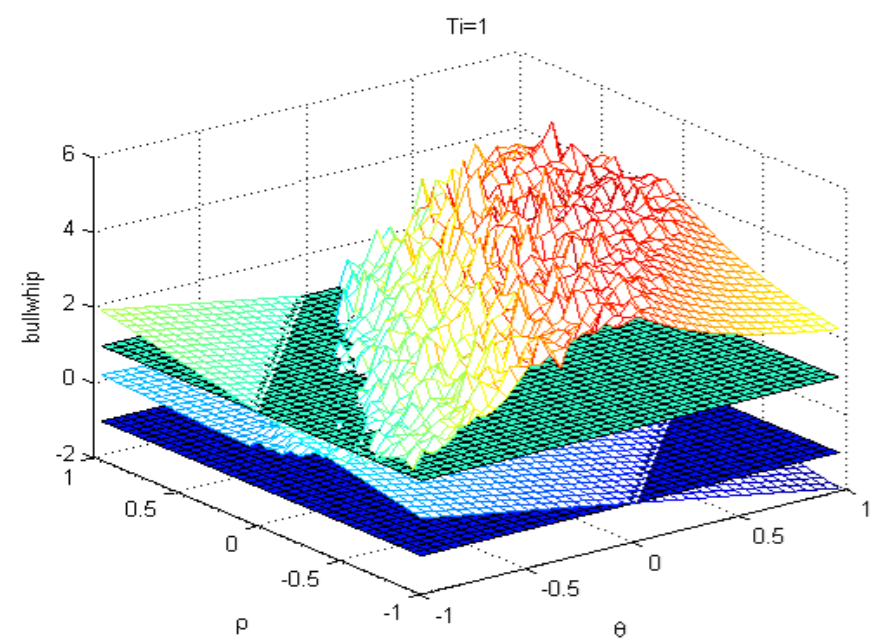

Fig. (2). Bullwhip surface (with noise) for $T_{i}=1$, Surfaces planes 1 (dark green) and -1 (dark blue) and the inclined plane $\rho-\theta$. 


\section{STABILITY ANALYSIS USING DIVERGENCE}

In this Section we will calculate the divergence of the supply chain given by Eq. (14) to develop a new type of control that is able of reducing the cumulative total costs and bullwhip. The divergence of a dynamical system is a local property defined as the trace of the Jacobian and then is given by:

$\operatorname{div}(J)=\rho-\theta-\frac{1}{T_{i}}+1$

The advantage in using the divergence to control the order policy resides in the fact that, in principle, it can be calculated using only the time series of the state variables: Orders, Net Stock and realized Demand as in [23-24], without the knowledge of the supply chain model. In addition, sometimes only one state variable is enough to calculate this value if it represents the dynamics of the system. The approach is based on the application of state space reconstruction techniques introduced in [29].

Here we have calculated the divergence using the model of the supply chain. The calculation of div using only temporal series of one measured variable of the system will be part of our future work but we believe that it is important to underline this aspect in order to understand the final objective of the present work.

\section{Divergence and Stability}

The divergence of the supply chain is a measure of the stability in the sense that it gives the rate of expansion or contraction of infinitesimal volume in the state space i.e. the volume that the states of the system can occupy, after one time step, starting from a given set of initial conditions.

When $T_{i}=1$ the divergence becomes the same as $\lambda_{2}$. Then asking for $|d i v|<1$ the stability condition is satisfied because all the eigenvalues are smaller than 1 in absolute value.

If $T_{i} \neq 1$ and $\lambda_{1}, \lambda_{2}, \lambda_{3}$ have the same sign, then $|\operatorname{div}|<1 \Rightarrow\left|\lambda_{1}\right|<1,\left|\lambda_{2}\right|<1,\left|\lambda_{3}\right|<1$, i.e. the stability condition is satisfied. Our objective is to check if $|\operatorname{div}|<1$ is a good strategy to control the bullwhip and reduce the costs in general.

\section{COSTS REDUCTION USING DIVERGENCE- BASED CONTROL}

To test the divergence control techniques we will concentrate in two particular values of $T_{i}: T_{i}=0.7$ and $T_{i}=2$. We have chosen these two values because the divergence plane cross the surface -1 and 1 for $T_{i}=0.7$ and $T_{i}=2$, respectively, as it can be seen in Fig. (3). In addition, it can be also observed that there is an increment of the total costs with the increase of the absolute value of divergence, div $=\rho-\theta+1-1 / T_{i}$, beyond the absolute value of one. In this figure we have represented the costs only for $-0.5<\rho, \theta<0.5$ instead for $-1<\rho, \theta<1$ because of their exponential increase beyond this region.
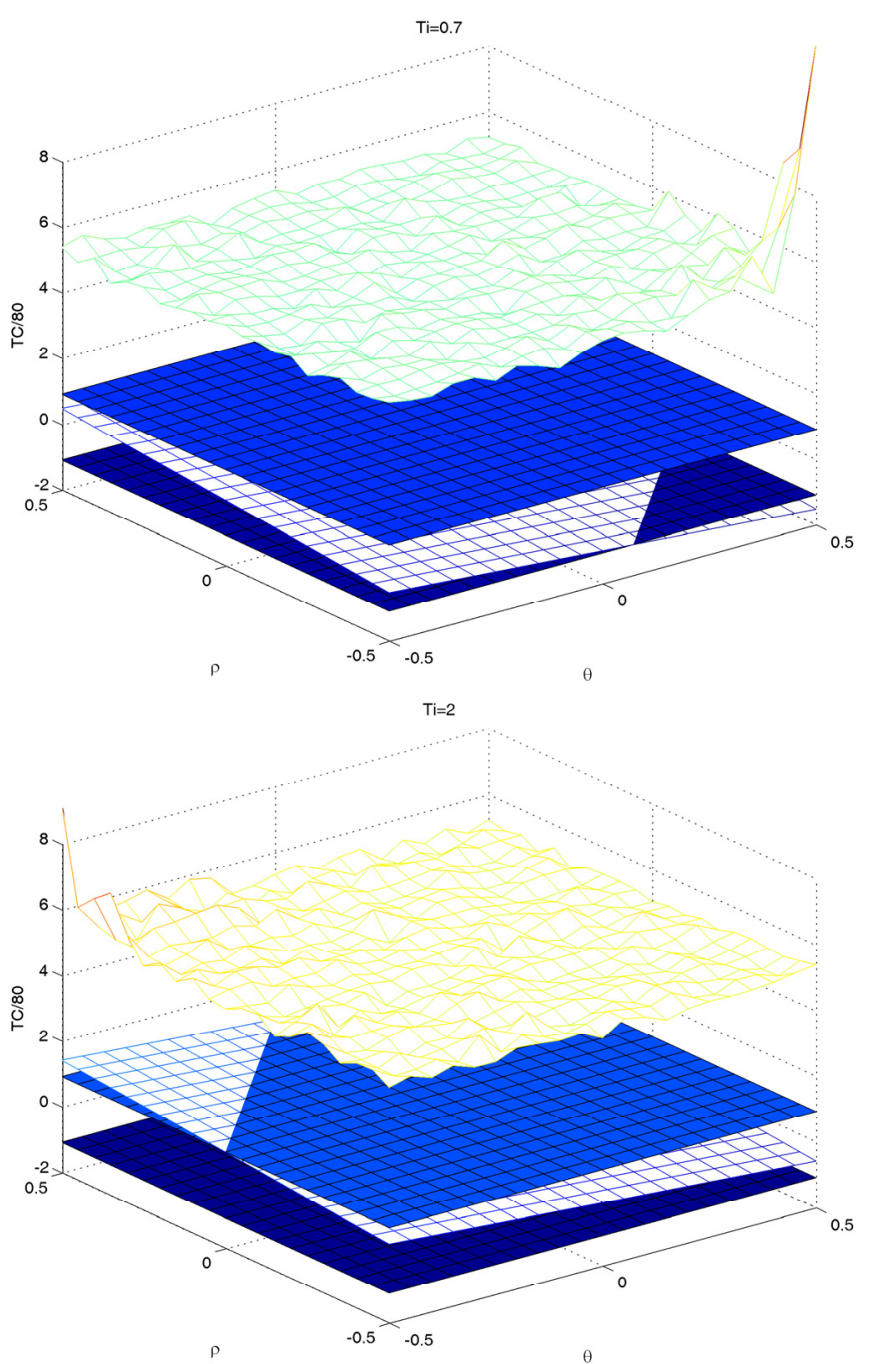

Fig. (3). Total costs (surface with noise) after 100 time units, surfaces planes 1 (light blue), -1 (dark blue), and $\rho-\theta$, respectively for $T_{i}=0.7, T_{i}=2$.

Now we are interested in checking if by acting on the parameter $T_{i}$, and maintaining divergence values in the range $[-1,1]$, the total costs will be reduced. The $T_{i}$ parameter is then modified only when divergence becomes bigger than 1 in absolute value in order to change the divergence value as follows:

if $\left|\operatorname{div}_{\mathrm{old}}\right| \geq 1$,

$d i v_{\text {new }}=\operatorname{div_{\text {old}}}-\operatorname{sign}\left(\operatorname{div_{\text {old}}}\right) \cdot\left(\left|d i v_{\text {old }}\right|-1\right)$

In practice, in order to obtain $\left|d i v_{n e w}\right|<1$ we subtract $\operatorname{sign}\left(\operatorname{div}_{\text {old }}\right) \cdot\left(\left|\operatorname{div}_{\text {old }}\right|-1\right)$ from $-1 / T_{i}$ as follows:

$\left(-1 / T_{i}\right)_{\text {new }}=\left(-1 / T_{i}\right)_{\text {old }}-\operatorname{sign}\left(\operatorname{div}_{\text {old }}\right) \cdot\left(\mid\right.$ div $\left._{\text {old }} \mid-1\right)$

and then

$\left(T_{i}\right)_{\text {mew }}=-\left[\left(-1 / T_{i}\right)_{\text {old }}-\operatorname{sign}\left(d i v_{\text {old }}\right)\left(\left|d i v_{\text {old }}\right|-1\right)\right]^{-1}$

With this calculation, we obtain a $T_{i}$ value that allows having a divergence smaller than 1 in absolute value. 
Graphically, the evolution of the controlled divergence with respect to the not controlled one is represented in Fig. (4).

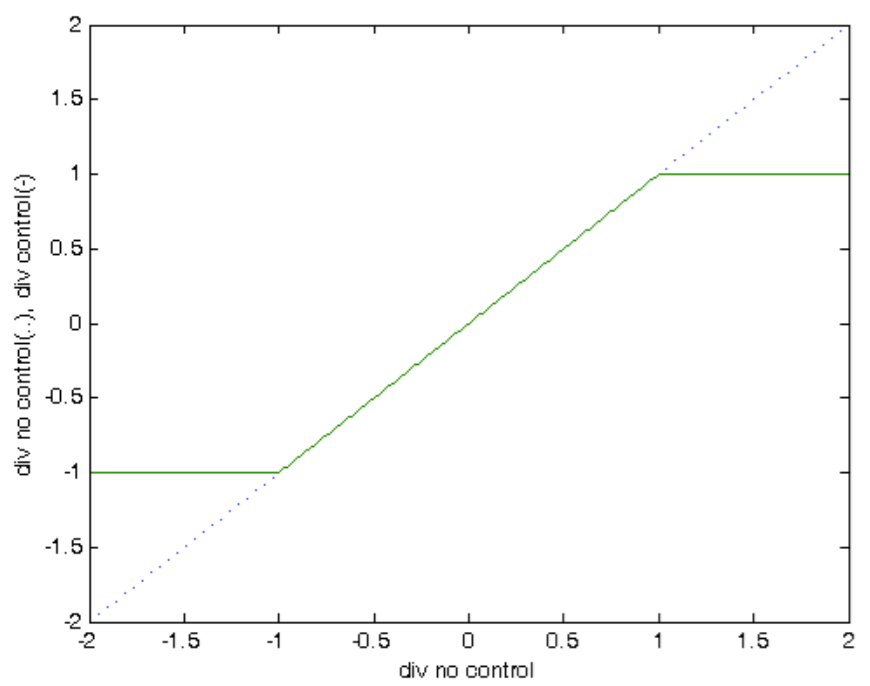

Fig. (4). Controlled (continuous) and non controlled divergence (dotted line) in respect to non controlled one in the interval $[-2,2]$.

Applying this kind of control divergence-based, it is possible to observe (Fig. 5) the reduction of the total costs surfaces for $T_{i}=0.7$ and $T_{i}=2$. This reduction occurs in correspondence with the divergence values that now stay inside the $[-1,1]$ interval.

To analyse how this type of control strategy affects the cumulative total costs over time, two particular cases are considered for which the divergence in absolute value is bigger than one and then the control will intervene modifying the results:

a) $\quad \mathrm{T}_{\mathrm{i}}=2, \rho=0.5, \theta=-0.5$

b) $\mathrm{T}_{\mathrm{i}}=0.7, \rho=-0.5, \theta=0.5$

In both cases (Figs. 6, 7), the advantage in applying divergence control is clear the cumulative total costs during the simulation period, defined as

$$
T C_{\text {cum }}=\sum_{t=1}^{n} T C_{t}
$$

are reduced. In order to quantify the reduction we may calculate the relative rate of increase of cumulative total costs, $R=\left[T C_{\text {cum }}(n)-T C_{\text {cum }}(1)\right] / T C_{\text {cum }}(1)$, with and without control. Without control $R$ is 0.48 for case a) and 1.5 in case b). Applying control we obtain $R=0.15$ in case a) and $R=0.41$ in case b) respectively (see Figs. 6, 7).

When the control is activated, the improvement in the costs occurs for all the parameters values, $\rho$ and $\theta$, (see Figs. 3,5 ), and not only for the special case represented.

We have analyzed the effects of changing the initial value of the control parameter $T_{i}$. The results are presented in Table 1. In the first column there are the $T_{i}$ value considered, in the second and third the logarithm of the mean total costs when the parameters $\rho$ and $\theta$ are varying between -1 and 1 with a step of 0.05 ; each case has been run during twenty time steps and twenty times in order to average the values obtained. In the last column, we have presented the percentage of the increment in the cumulative total costs $\left(T C_{\text {cum }}\right)$ that is obtained if we do not apply the divergence control strategy. By considering all cases we can observe that in average they are reduced approximately by $63 \%$ applying our control strategy. In particular, for low $T_{i}$ values the reduction in $T C_{c u m}$ is lower than for high $T_{i}$ values, and after a fast increase, until $T_{i}=2.5$, the values tend to oscillate around $65 \%$ approximately. It should be noticed that when $|d i v|<1$ no control action is performed and therefore, as $T_{i}$ increases the actuation of the control algorithm are more frequent, see Eq. (18).
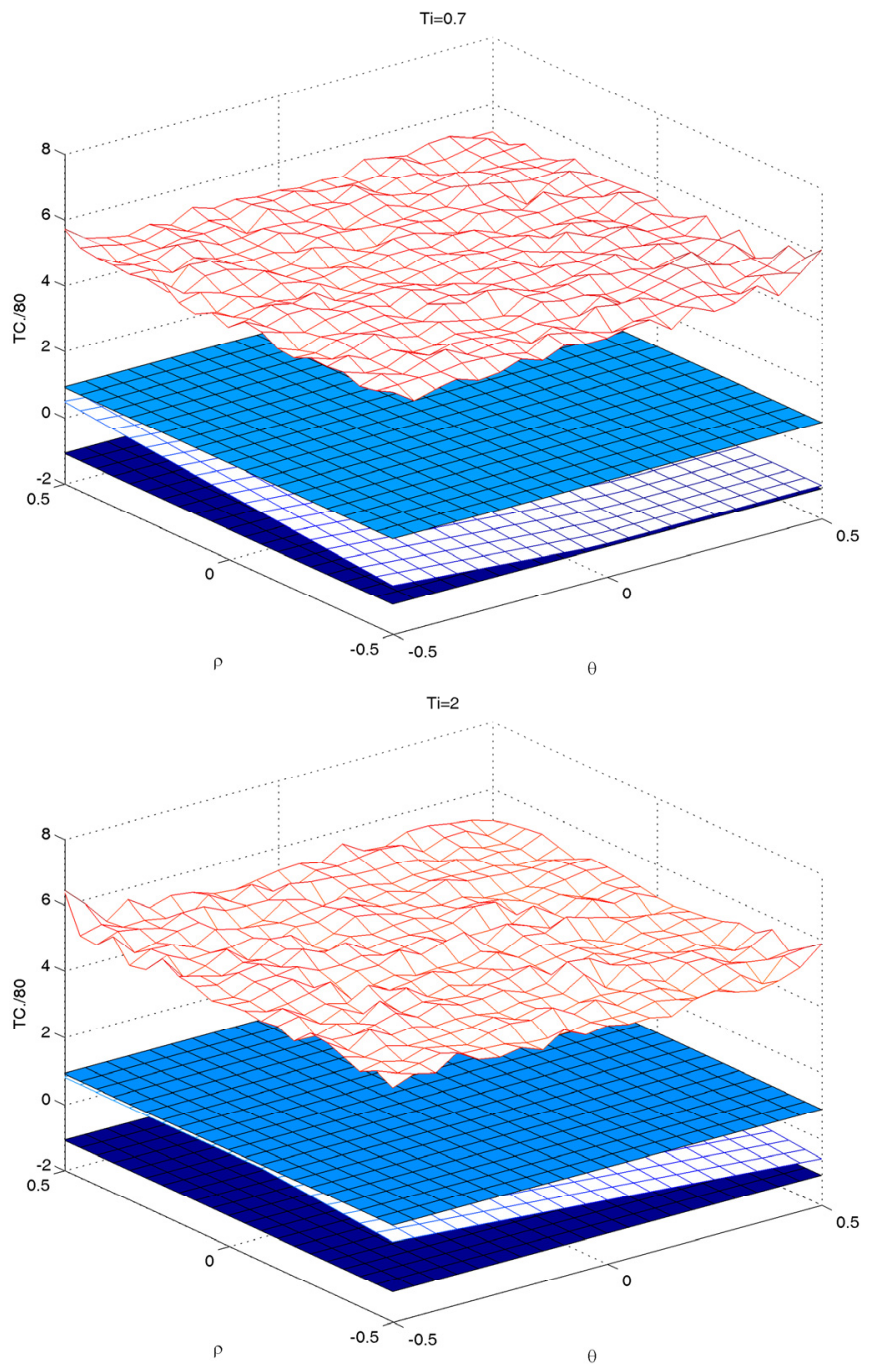

Fig. (5). Controlled divergence total costs (surface with noise) after 100 time units, surfaces planes 1 (light blue), -1 (dark blue), and $\rho$ $\theta$, respectively for $T_{i}=0.7, T_{i}=2$.

\section{BULLWHIP REDUCTION USING DIVERGENCE- BASED CONTROL}

As it has been discussed previously, the control strategy based on maintaining the absolute value of the divergence of the supply chain between $[-1,1]$, is able to reduce the costs. Let us now analyse what are the effects on the bullwhip values. In Fig. (8), we can see the bullwhip surface for $T_{i}=0.7$ without and with divergence control. If one observes the part 
of the parameter space, in correspondence with high $\theta$ values (near to 1 ) and small $\rho$ values (near to -1 ), it is possible to see how the activation of the control implies a reduction of the bullwhip effect. These parameters values correspond to an order policy that give more importance to the error between real and forecast demand than to the deviation of forecasted demand from the mean value. For the other parameter values the bullwhip effect reduction does not occur. In the middle of parameters space where $|d i v|<1$ the divergence control is not activated. In the other part of parameter's space where $|d i v|>1$, small $\theta$ values and high $\rho$ values, the control is also activated but the bullwhip is not reduced. Nevertheless, in this case, as it can be seen from Fig. (8), the bullwhip is very small even without control.
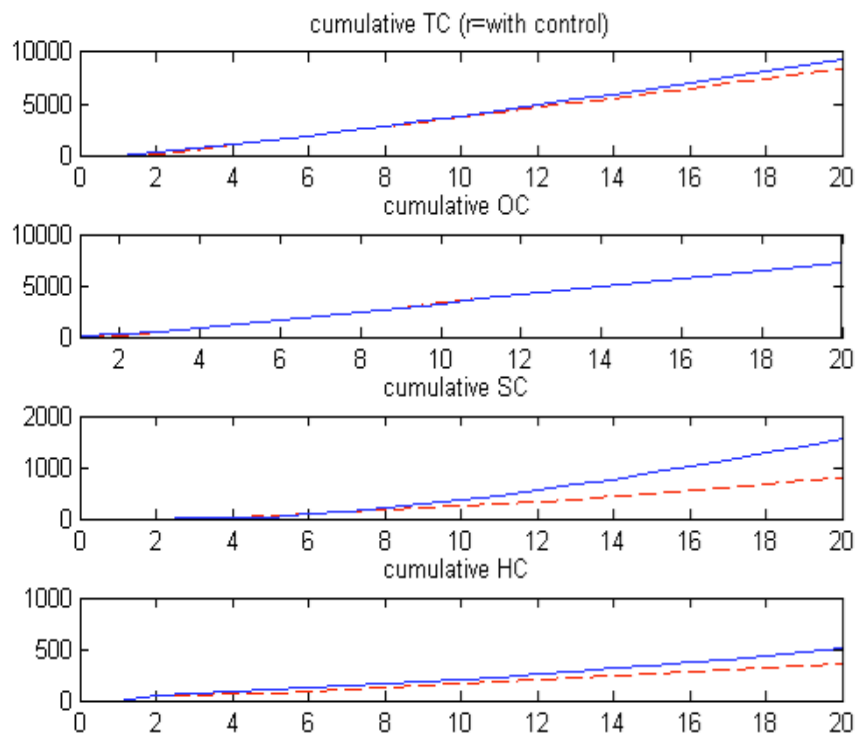

Fig. (6). Costs without (continuous line) and with (dot line) divergence control in time, case a) $T_{i}=2, \rho=0.5, \theta=-0.5$. Costs reduction: $\mathrm{R}$ without control - $\mathrm{R}$ with control $=0.33$.
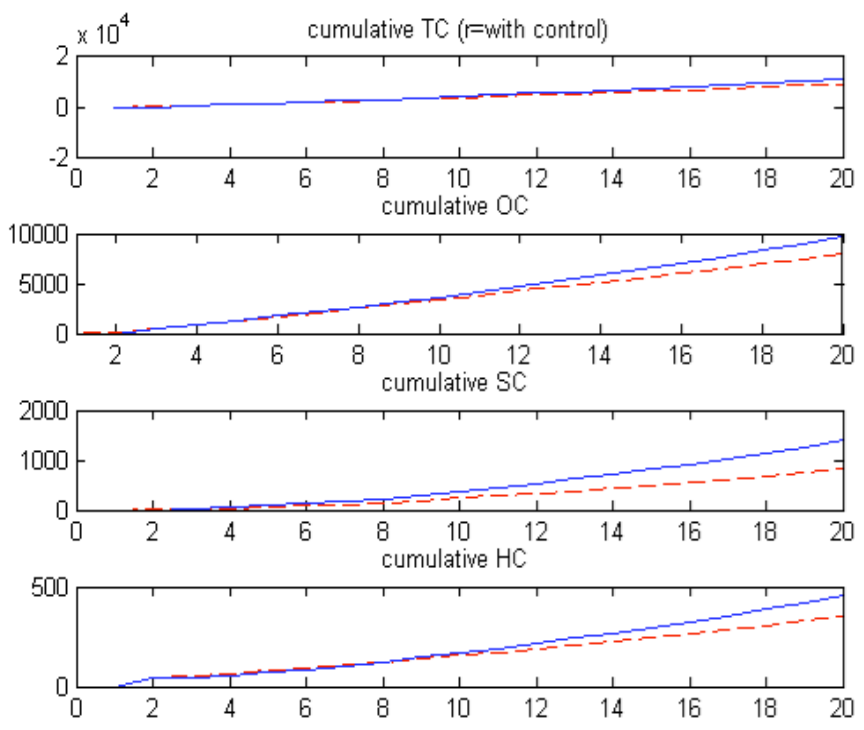

Fig. (7). Costs without (continuous line) and with (dot line) divergence control in time, case b): $T_{i}=0.7 \rho=-0.5, \theta=0.5$. Costs reduction: $\mathrm{R}$ without control - $\mathrm{R}$ with control=1.09.
Table 1. Logarithm of the Mean Value of Total Costs for Different $T_{i}$ Values, when $\rho$ and $\theta$ are Varying Between -1 and 1 with an Step of 0.05 , without $\left(T C_{c u m}^{n c}\right)$ and with ( $T C_{c u m}^{c}$ ) Divergence Control for 20 Simulation Runs each Combination of Parameters

\begin{tabular}{|c|c|c|c|}
\hline $\mathbf{T}_{\mathbf{i}}$ & $T C_{\text {cum }}^{n c}$ & $T C_{\text {cum }}^{c}$ & Gain (\%) \\
\hline 0.5 & 10.7734 & 10.7083 & 6.3026 \\
\hline 1.0 & 11.5877 & 10.9883 & 45.0859 \\
\hline 1.5 & 11.5993 & 10.8698 & 51.7850 \\
\hline 2.0 & 11.7926 & 11.0314 & 53.2894 \\
\hline 2.5 & 11.9066 & 10.8179 & 66.3346 \\
\hline 3.0 & 11.9005 & 10.9408 & 61.6992 \\
\hline 3.5 & 11.8798 & 11.0365 & 56.9712 \\
\hline 4.0 & 12.1444 & 11.3136 & 56.4299 \\
\hline 4.5 & 12.1076 & 11.2705 & 56.7036 \\
\hline 5.0 & 12.1493 & 11.0166 & 67.7838 \\
\hline 5.5 & 12.1466 & 11.2143 & 60.6353 \\
\hline 6.0 & 11.9636 & 11.2891 & 49.0589 \\
\hline 6.5 & 12.1481 & 10.9644 & 69.3856 \\
\hline 7.0 & 11.9673 & 10.9451 & 64.0197 \\
\hline 7.5 & 12.0793 & 11.1492 & 60.5486 \\
\hline 8.0 & 12.1676 & 10.9176 & 71.3495 \\
\hline 8.5 & 12.0981 & 10.9855 & 67.1297 \\
\hline 9.0 & 12.1813 & 10.7704 & 75.6076 \\
\hline 9.5 & 12.3316 & 11.3031 & 64.2457 \\
\hline 10.0 & 11.8306 & 10.6557 & 69.1150 \\
\hline 10.5 & 11.9813 & 11.0265 & 61.5111 \\
\hline 11.0 & 12.0294 & 11.1342 & 59.1474 \\
\hline 11.5 & 12.1204 & 10.8850 & 70.9282 \\
\hline 12.0 & 12.2081 & 11.2591 & 61.2872 \\
\hline 12.5 & 12.4134 & 11.0014 & 75.6345 \\
\hline 13.0 & 11.9585 & 11.0297 & 60.4973 \\
\hline 13.5 & 12.2391 & 11.3215 & 60.0523 \\
\hline 14.0 & 12.2050 & 10.9544 & 71.3667 \\
\hline 14.5 & 11.5667 & 10.8630 & 50.5249 \\
\hline 15.0 & 12.4562 & 11.0025 & 76.6296 \\
\hline 15.5 & 12.1701 & 10.9530 & 70.3912 \\
\hline 16.0 & 11.9659 & 10.9846 & 62.5176 \\
\hline 16.5 & 12.3291 & 11.0453 & 72.3017 \\
\hline 17.0 & 12.2331 & 10.8312 & 75.3871 \\
\hline 17.5 & 12.2027 & 10.9775 & 70.6301 \\
\hline 18.0 & 12.1794 & 11.1155 & 65.4893 \\
\hline 18.5 & 12.2126 & 11.1991 & 63.7054 \\
\hline 19.0 & 12.1499 & 11.0093 & 68.0373 \\
\hline 19.5 & 12.3574 & 11.2053 & 68.4027 \\
\hline 20.0 & 12.1506 & 11.0727 & 65.9691 \\
\hline
\end{tabular}



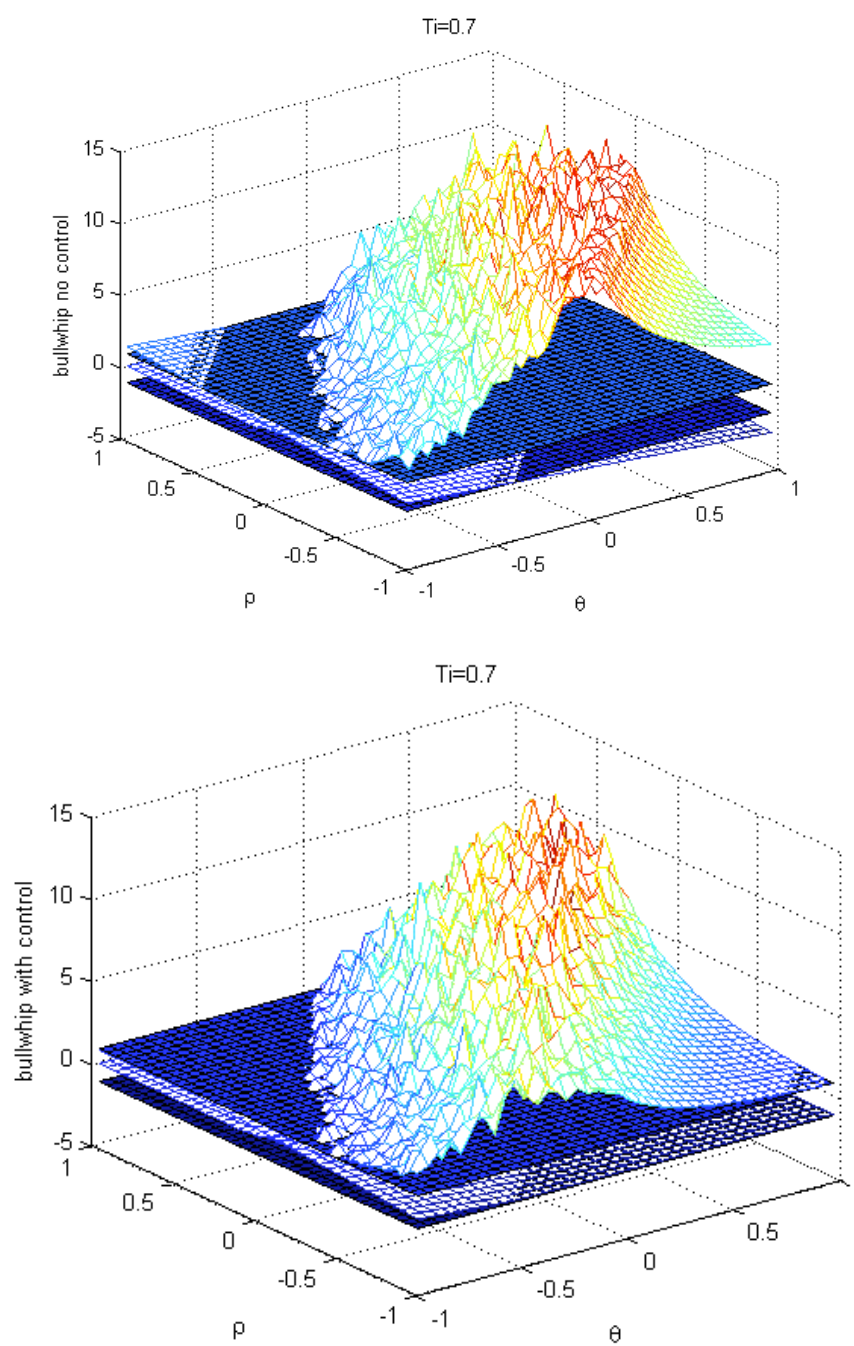

Fig. (8). Bullwhip surface without and with divergence control for $T_{i}=0.7$.

If we consider the other value of the control parameter, $T_{i}=2$, see Fig. (9), we can observe a similar behaviour. In this case, perhaps because the control acts in a region where the bullwhip surface is more smooth (high $\theta$ values and small $\rho$ values), it is even more clear the action of the control on the reduction of the bullwhip. In fact a clear discontinuity in the bullwhip slope is observed in Fig. (9). The results for different $T_{i}$ values as a function of $\rho$ and $\theta$ are presented in Table 2. Similarly to Table $\mathbf{1}$, in Table 2 the results correspond to 20 time steps and each combination of parameters was run 20 times and averaged. In the second and third column there are mean bullwhip values without and with control. In the third column the percentage of reduction applying control indicate that we can have a strong reduction only for certain values of $T_{i}$, whereas for the others we obtain similar values. Applying the control strategy there is a strong reduction of bullwhip for $T_{i}$ values from 0.5 until 2 then the difference in bullwhip with or without control becomes nearly zero.

Comparing Tables $\mathbf{1}$ and $\mathbf{2}$, we can observe that in the interval of $T_{i}$ values between 0.5 and 2 , by applying the control strategy based on divergence we have both bullwhip reduction and a decreasing of cumulative total costs. For $T_{i}$ higher than 2 the gain applying the control strategy remains high but there is no a bullwhip reduction. High $T_{i}$ values imply that the order policy without control does not consider the difference between the net stock and the real demand variance, see Eq. (10).
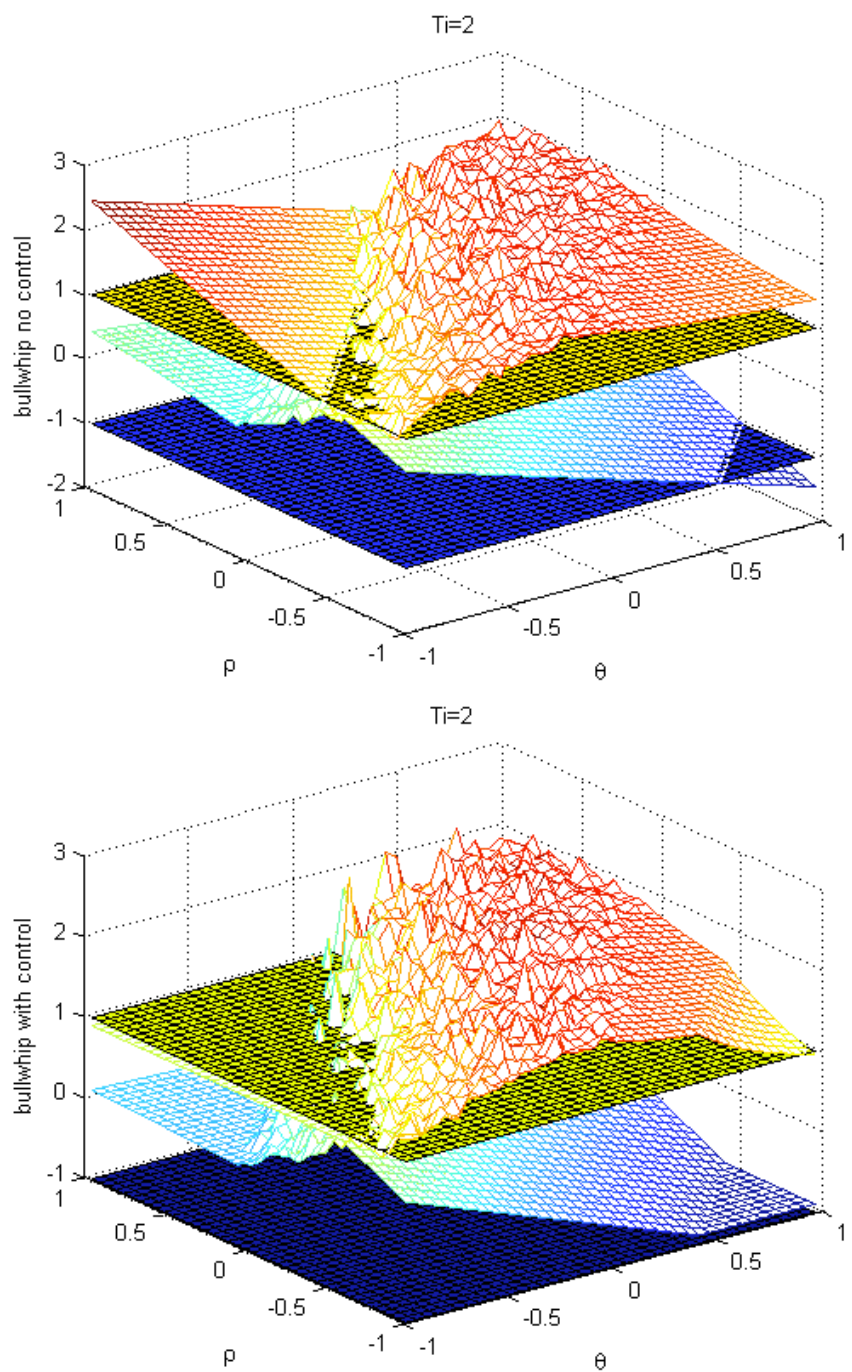

Fig. (9). Bullwhip surface without and with divergence control for $T_{i}=2.0$.

\section{CONCLUSIONS AND FUTURE WORK}

In this work we have considered, following [1, 5] a single-product one echelon supply chain with a zero replenishment lead time and only a single, order-of events review period (see Fig. 1) in which an Order-Up-To (OUT) order policy is applied and the demand is forecasted using an ARMA model, Eq. (13). Using this model the relationships between bullwhip and stability have been analysed, as well as the relationships between the divergence based criterion and stability conditions.

By comparing bullwhip and stability surface (see Fig. 2) in the $(\rho, \theta)$ plane we have found that the stability region contains the parameter values for which the bullwhip surface is equal to one, but, on the other side, small bullwhip values can be obtained even in the instability region, i.e. when the surface $\rho-\theta$ has values higher than 1 or smaller than -1 . 
Table 2. Mean Values of Bullwhip for Different $T_{i}$ Values - when $\rho$ and $\theta$ are Varying Between $\mathbf{- 0 . 5}$ and $\mathbf{- 1}$ and 0.5 and 1 , Respectively- without $\left(B_{n c}\right)$ and with $\left(B_{c}\right)$ Divergence Control and its Differences $\left(\left(B_{n c}-B_{c}\right) \cdot 100 / B_{n c}\right)$ in Percentage for 20 Simulation Runs

\begin{tabular}{|c|c|c|c|}
\hline$T_{i}$ & $\boldsymbol{B}_{n c}$ & $\boldsymbol{B}_{c}$ & Diff. (\%) \\
\hline 0.5 & 2.8237 & 1.2959 & 54.1063 \\
\hline 1.0 & 1.5449 & 1.2574 & 18.6096 \\
\hline 1.5 & 1.3292 & 1.2341 & 7.1547 \\
\hline 2.0 & 1.1685 & 1.1634 & 0.4365 \\
\hline 2.5 & 1.1378 & 1.1230 & 1.3008 \\
\hline 3.0 & 1.0886 & 1.1028 & -1.3044 \\
\hline 3.5 & 1.0799 & 1.0722 & 0.7130 \\
\hline 4.0 & 1.0533 & 1.0656 & -1.1678 \\
\hline 4.5 & 1.0343 & 1.0432 & -0.8605 \\
\hline 5.0 & 1.0308 & 1.0391 & -0.8052 \\
\hline 5.5 & 1.0270 & 1.0295 & -0.2434 \\
\hline 6.0 & 1.0245 & 1.0235 & 0.0976 \\
\hline 6.5 & 1.0142 & 1.0205 & -0.6212 \\
\hline 7.0 & 1.0118 & 1.0200 & -0.8104 \\
\hline 7.5 & 1.0135 & 1.0149 & -0.1381 \\
\hline 8.0 & 1.0031 & 1.0129 & -0.9770 \\
\hline 8.5 & 1.0096 & 1.0105 & -0.0891 \\
\hline 9.0 & 1.0061 & 1.0074 & -0.1292 \\
\hline 9.5 & 1.0076 & 1.0100 & -0.2382 \\
\hline 10.0 & 1.0021 & 1.0047 & -0.2595 \\
\hline 10.5 & 1.0004 & 1.0027 & -0.2299 \\
\hline 11.0 & 1.0023 & 1.0067 & -0.4390 \\
\hline 11.5 & 0.9998 & 1.0021 & -0.2300 \\
\hline 12.0 & 1.0006 & 1.0058 & -0.5197 \\
\hline 12.5 & 1.0003 & 1.0011 & -0.0800 \\
\hline 13.0 & 0.9989 & 1.0014 & -0.2503 \\
\hline 13.5 & 0.9999 & 0.9997 & 0.0200 \\
\hline 14.0 & 1.0003 & 1.0044 & -0.4099 \\
\hline 14.5 & 1.0005 & 1.0009 & -0.0400 \\
\hline 15.0 & 0.9995 & 0.9993 & 0.0200 \\
\hline 15.5 & 0.9986 & 1.0010 & -0.2403 \\
\hline 16.0 & 1.0002 & 1.0001 & 0.0100 \\
\hline 16.5 & 0.9978 & 0.9995 & -0.1704 \\
\hline 17.0 & 0.9972 & 1.0001 & -0.2908 \\
\hline 17.5 & 1.0005 & 1.0001 & 0.0400 \\
\hline 18.0 & 0.9988 & 0.9998 & -0.1001 \\
\hline 18.5 & 0.9962 & 0.9995 & -0.3313 \\
\hline 19.0 & 0.9996 & 0.9976 & 0.2001 \\
\hline 19.5 & 0.9996 & 0.9994 & 0.0200 \\
\hline 20.0 & 0.9972 & 0.9978 & -0.0602 \\
\hline
\end{tabular}

The divergence criterion, $|d i v|<1$, is introduced in order to reduce bullwhip and costs by decreasing the instability of the supply chain. For some values of control parameters, $T_{i}=1$, it was showed that checking $|d i v|<1$ is identical to check the stability of the system using its eigenvalues. It was found that (Fig. 3) in correspondence of the values of $\rho$ and $\theta$ for which the divergence is bigger than one in absolute value, the total costs increase. If the divergence values are controlled by changing the control parameter $T_{i}$ in order to maintain $|d i v|<1$ we obtain a substantial reduction of the total costs (Fig. 5).

In the two particular cases analyzed, the advantages of the application of divergence control are checked by measuring the cumulative total costs in correspondence of some parameters values for which we are sure that the control is applied, see Figs. (6) and (7). In both cases, a reduction of the cumulative total costs of more than $30 \%$ is obtained. In addition, as it can be seen in Figs. (8) and (9), the control using divergence allows a bullwhip reduction too for some of the $T_{i}$ values considered. This is clear in the region of $\rho<0$ and $\theta>0$. When $\rho>0$ and $\theta<0$, even though there is a reduction of costs, this is not accompanied by a reduction in the bullwhip, but the bullwhip is already small in this region. The extension of these previous results to a general set of parameters can be seen in Tables $\mathbf{1}$ and $\mathbf{2}$. In these cases we can observe that this control strategy always allows a decreasing of cumulative Total Costs but not of bullwhip effect. Anyway, as it is well known, the bullwhip along cannot be considered in general an indication of the performance of a supply chain.

As it was pointed out in [21], the functioning of the proportional controller depends on the demand model and its parameter values and care must be taken before implementing it on real-life situations. On the contrary, using the divergence the control parameter, $T_{i}$, adapts continuously, avoiding $|d i v| \geq 1$. In addition, the major advantage in using divergence is that, in principle, it can be calculated using only the time series of one of the variables without the model equations [23-24] and, therefore, it should be possible to extent it to real-life situations. Our future work will aim at developing an efficient algorithm for on-line control of supply chains without the need of forecasting the demand, based on state space reconstruction and divergence estimation.

\section{ACKNOWLEDGEMENTS}

The authors gratefully acknowledge the financial support of the European Commission DG RTD funded MANMADE NEST Project (Contract No 043363) and Fondazione Cariplo.

\section{REFERENCES}

[1] Chen YF, Disney SM. The order-up-to policy "sweet spot" - Using proportional controllers to eliminate the bullwhip problem, in EUROMA POMS Conference, Como Lake, Italy 2003; 2: 551-60.

[2] Lee HL, Padmanabhan V, Whang S. Information distortion in the Supply Chain: The Bullwhip Effect. Manage Sci 1997; 43: 546-58.

[3] Carlsson C, Fuller R. Reducing the bullwhip effect by means of intelligent, soft computing methods, Proceedings of the $34^{\text {th }} \mathrm{Ha}$ waii, International Conference on System Sciences 2001; 1-10. 
[4] Geary S, Disney SM, Towill DR. On bullwhip in supply chainshistorical review, present practice and expected future impact. Int J Prod Econ 2006; 101: 2-18.

[5] Chen YF, Disney SM. The myopic order-up-to policy with a feedback controller. Int J Prod Res 2007; 45: 351-68.

[6] Forrester JW. Industrial Dynamics. MIT Press, Cambridge, MS, 1961.

[7] Sterman JD. Modeling managerial behavior: Misperceptions of feedback in a dynamic decision-making experiment. Manage Sci 1989; 35: 321-39.

[8] Lee H. Information distortion in supply chain: the bullwhip effect. Manage Sci 1997; 43: 546-58

[9] Lee HL, Padmanabhan V, Whang S. The bullwhip effect in supply chains. Sloan Manage Rev 1997; 38: 93-102.

[10] Metters R. Quantifying the bullwhip effect in supply chains. J Oper Manage 1997; 15: 89-100.

[11] Towill DR, Del Vecchio A. The application of filter theory to the study of supply chain dynamics. Prod Plan Control 1994; 5: 82-96.

[12] Makajic-Nikolic D, Panic B, Vujosevic M. Bullwhip effect and supply chain modelling and analysis using CPN tools, In: Kurt Jensen, Ed. Proceedings of the fifth workshop and tutorial on practical use of coloured petri nets and the CPN tools, Aarhus, Denmark, DAIMI PB - 570, October 8-11, 2004; 219-34.

[13] Sudhir JRD, Chandrasekharan R. A simulation-based genetic algorithm for inventory optimization in a serial supply chain. Int Trans Oper Res 2005; 2: 101-27.

[14] Strozzi F, Bosch J, Zaldívar JM. Beer Game order policy optimization under changing customer demand. Decis Support Syst 2007; 42: 2153-63.

[15] Chen F, Drezner Z, Ryan JK, Simki-Levi D. Quantifying the bullwhip effect in a simple supply chain: the impact of forecasting, lead time, and information. Manage Sci 2000; 46: 436-43.
[16] Helbing D, Lammer S. http: //arxiv.org/abs/cond--mat/0411486. 2006

[17] Kleijnen JPC. Supply Chain simulation: a survey. Int J Sim Proc Mod 2005; 1: 82-9.

[18] Thomsen JS, Mosekilde E, Sterman JD. Hyperchaotic phenomena in dynamic decision making. J Syst An Mod Sim 1992; 9: 137-56.

[19] Laugesen J, Mosekilde E. Border-collision bifurcations in dynamic management game. Comp Oper Res 2006; 33: 464-78.

[20] Sarimveis H, Patrinos P, Tarantilis CD, Kiranoudis CT. Dynamic modeling and control of supply chain systems: A review. Comp Oper Res 2008; 35: 3530-61.

[21] Gaalman G, Disney SM. State space investigation of the bullwhip problem with ARMA $(1,1)$ demand process. Int J Prod Econ 2006 104: 327-39.

[22] Tzafestas S, Kapsiotis G, Kyriannakis E. Model-based predictive control for generalized production planning problems. Comp Indust 1997; 34: 201-10.

[23] Bosch J, Kerr D, Snee TJ, Strozzi F, Zaldívar JM. Runaway detection in a pilot plant facility. Ind Eng Chem Res 2004; 43: 7019-24.

[24] Zaldívar JM, Strozzi F. Time-step volume-preserving control of chemical reactors. Open Chem Eng J 2007; 1: 8-16.

[25] Chacon G, Terwiesch C. An Introduction to Operations Management. McGraw Hill: Irwin 2006.

[26] Disney SM, Farasyn I, Lambrecht MR, Towill DR, Van De Velde W. Taming bullwhip whilst watching customer service. Eur J Oper Res 2006; 173: 151-72.

[27] Gilbert K. An ARIMA supply chain model. Manage Sci 2005; 51: 305-10.

[28] Alwyn LC. Bullwhip realities. Proceedings of the INFORMS Conference. Miami Beach, USA. November $4^{\text {th }}-7^{\text {th }}, 2001$.

[29] Packard N, Crutchfield J, Farmer D, Shaw R. Geometry from a time series. Phys Rev Lett 1980; 45: 712-5.

Received: July 25, 2008

(C) Strozzi et al.; Licensee Bentham Open.

This is an open access article licensed under the terms of the Creative Commons Attribution Non-Commercial License (http://creativecommons.org/licenses/by$\mathrm{nc} / 3.0 /$ ) which permits unrestricted, non-commercial use, distribution and reproduction in any medium, provided the work is properly cited. 